\title{
Reply to: "Requirements for applying a case-control study model and clinical significance of changes in the visual analogue scale score in abdominal pain"
}

\author{
Graziano Ceccarelli $\cdot$ Luciano Casciola $\cdot$ Massimo Codacci-Pisanelli \\ Alberto Bartoli · Lelio Di Zitti · Alessandro Spaziani · \\ Alessia Biancafarina $\cdot$ Massimo Stefanoni · Alberto Patriti
}

Published online: 14 May 2009

(C) Springer Science+Business Media, LLC 2009

We greatly appreciated the detailed epidemiologic explanation of a case-control study but we do not believe that our study design biases the results presented.

One of their arguments was that reduced pain and bleeding at the trocar site in the fibrin sealant group was not related to the different mesh fixation technique but only to the laparoscopic procedure.

On the contrary, the use of fibrin sealant marks a major difference between the two techniques that explains the statistical differences in terms of postoperative pain and bleeding. The fibrin sealant device may be introduced only through a 5-mm trocar on the right flank instead of a 10$\mathrm{mm}$ trocar required by a stapling device. In the staple technique we have to suture the fascial defect of all 10-mm trocar sites to avoid postoperative incisional hernias [1], as recommended by several authors.

Unfortunately, this practice is known to determine pain or discomfort during the first postoperative week, as frequently observed also in cases of laparoscopic repair of incisional hernias with mesh fixation by fascial suture [2], but the pain generally disappears after the first month. So,

We would like to thank Dr. Zonta and colleagues for their interesting suggestions and remarks on our paper.

G. Ceccarelli · L. Casciola - A. Bartoli .

L. Di Zitti - A. Spaziani - A. Biancafarina - M. Stefanoni ·

A. Patriti

Department of General, Vascular and Mininvasive Surgery,

Spoleto Hospital, Spoleto, Italy

M. Codacci-Pisanelli ( $\square)$

Surgical Department "Pietro Valdoni",

University of Rome "La Sapienza", Roma, Italy

e-mail: massimo.codacci@uniroma1.it in our long experience of 16 years and around 4,000 laparoscopic hernia repair using transabdominal preperitoneal (TAPP) technique, we have encountered only two cases $(0.05 \%)$ of chronic pain.

Moreover, it is commonly noted that $10-\mathrm{mm}$ port sites are more prone to postoperative bleeding than are $5-\mathrm{mm}$ ones.

Second, the visual analogue scale (VAS) is one of the most commonly used methods of measuring postoperative pain. Of course, VAS consists of a $10-\mathrm{cm}$ line anchored by two extremes of pain; patients are asked to make a mark on the line that represents their level of perceived pain intensity. (Findings suggest that ratings of $0-4 \mathrm{~mm}$ can be considered pain free, and 5-44 mm as mild pain [2].) In keeping with current literature, we determine as positive (i.e., with pain) the cases of patients that placed their mark above 4 (i.e., $4 \mathrm{~cm}$ ).

This statistical test was correctly used in our study to compare the patient pain of the two groups and we believe that there are no convincing reasons that make other analytical methods proposed superior to or more accurate than this one [3].

\section{References}

1. Morrison CP, Wemyss-Holden SA, Iswariah H, Maddern GJ (2002) Lateral laparoscopic port sites should all be closed: the incisional "spigelian" hernia. Surg Endosc 16(9):1364

2. LeBlanc KA (2007) Laparoscopic incisional hernia repair: are transfascial sutures necessary? A review of the literature. Surg Endosc 21(4):508-513

3. Lee EO, Jung MS (1988) A study for assessing accuracy rates of pain rating scales (KPRS and VAS). Kanho Hakhoe Chi 18(3): 239-244 